\title{
Una nueva oficina en el liceo: la instalación de los orientadores como política educacional en Chile (c.1946-c.1953)*
}

\section{Pablo Andrés Toro Blanco}

Afiliado institucionalmente con la Universidad Alberto Hurtado (Chile).Correo electrónico: ptoro@ uahurtado.cl. El autor es doctor en Historia de la Pontificia Universidad Católica de Chile. Entre sus temas de interés están Historia de la educación en Chile, Historia de los movimientos estudiantiles en Chile, Historia de las emociones e Historia de la Educación.

Recibido: 5 de agosto de 2017

Aprobado: 12 de diciembre de 2017

Modificado: 1 de enero de 2018

Artículo de investigación científica

DOI: http://dx.doi.org/10.15648/hc.33.2018.11

Este artículo forma parte del proyecto: "Lo que se vive y lo que debe ser vivido: discusiones y prácticas de educación emocional de los estudiantes secundarios en Chile, c.1912-c.1964” Financiado por Fondecyt Regular 1140604 (Chile).

Esta publicación está bajo una licencia Creative Commons Reconocimiento-NoComercial 4.0 
Una nueva oficina en el liceo: la instalación de los orientadores como política educacional en Chile (c.1946-c.1953)

\title{
Resumen
}

En el contexto de la internacionalización de las políticas educativas a partir de la posguerra, la orientación escolar fue un campo emergente en las reformas emprendidas en la enseñanza secundaria en Chile. Fue entendida como un espacio con una doble función. Por un lado, debía guiar a los estudiantes hacia decisiones vocacionales acordes a las necesidades del país. Además, le correspondía inducir en ellos procesos de conocimiento y regulación emocional. En este texto se abordan, sintéticamente, algunos aspectos de su instalación en los liceos y se caracterizan algunos de sus desafíos, especialmente respecto a la segunda función señalada.

Palabras clave: historia de la educación chilena, orientación escolar, historia transnacional, historia de las emociones.

\section{A new office in the school (liceo): The establishment of counselors as an educa- tional policy in Chile (c.1946-c.1953)}

\begin{abstract}
In the context of the internationalization of educational policies after the Second World War, school counselling was an emerging field in the reforms undertaken in secondary education in Chile. It was understood as a realm with a double function. On the one hand, it had to guide students towards vocational decisions according to the needs of the country. In addition, it should induce processes of knowledge and emotional regulation in students. In this paper, it is synthetically addressed some aspects of the establishment of school counselors in the Lyceums as well as some of its challenges, especially regarding the second mentioned function.
\end{abstract}

Keywords: history of Chilean education, school counselling, transnational history, history of emotions.

Um novo escritório no liceo: a instalação dos orientadores como política educacional em Chile (c.1946-c.1953)

\section{Resumo}

No contexto da internacionalización das políticas educativas a partir da posguerra, a 
orientação escolar foi um campo emergente nas reformas empreendidas no ensino secundário em Chile. Foi entendida como um espaço com uma dupla função. Por um lado, devia guiar aos estudantes para decisões vocacionales conformes às necessidades do país. Ademais, correspondia-lhe induzir neles processos de conhecimento e regulação emocional. Neste texto aborda-se, sinteticamente, alguns aspectos de sua instalação nos liceos e caracterizam-se alguns de seus desafios, especialmente com respeito à segunda função assinalada.

Palavras-chave: história da educação chilena, orientação escolar, história multinacional, história das emoções.

\section{Un nouveau bureau dans le lycée: l'installation des conseillers scolaires comme politique éducative au Chili (c. 1946-c.1953)}

\section{Résumé}

Dans le contexte de l'internationalisation des politiques éducatives à partir de la post guerre, l'orientation scolaire a été un domaine émergent à l'égard des réformes adoptées dans l'enseignement secondaire au Chili. Celle-ci a été comprise comme un espace d'une double fonctionne. D’un côté, le conseiller scolaire devait guider les élèves vers des choix vocationnelles en accord avec les besoins du pays. De l'autre, il devait les induire vers des processus de connaissance et régulation émotionnelle. Dans ce texte ils sont abordés synthétiquement, quelques aspects de son installation dans les lycées et quelques-uns de ses défis y sont caractérisés, en particulier concernant la deuxième fonctionne précédemment signalée.

Mots clés : histoire de l'éducation chilienne, orientation scolaire, histoire transnational, histoire des émotions.

\section{INTRODUCCIÓN}

A fines de 1918 llegaba una carta desde Estados Unidos al hogar del doctor Carlos Fernández Peña, Presidente de la Asociación de Educación Nacional (AEN) y militante comprometido con la causa de la obligatoriedad de la instrucción primaria en Chile que, finalmente, se instauraría en 1920. Con el entusiasmo de quien se encuentra en una misión formativa en uno 
de los focos de irradiación de nuevas orientaciones educativas en esos tiempos, el profesor Max Salas Marchán, desde el frío invierno de Nueva York, compartía con su compañero de luchas gremiales y académicas su experiencia respecto a la actitud de los profesores norteamericanos frente a sus alumnos. Indicaba Salas que, durante una visita a una escuela, al acercarse a felicitar a una profesora

"ella me contestó sencillamente: "yo los ayudo todo lo que puedo". Esa frase me impresionó como una revelación: Ella no dijo: "yo les enseño, les exijo, cumplo el programa, adopto un buen texto..." Sin perjuicio de hacer esto mismo, ella modifica su actitud de maestra, contemplando, de preferencia a cualquiera otra cosa, el interés y bienestar de sus alumnos. "Yo los ayudo" significa: "yo los amo y procuro comprenderlos". Y aquí está la esencia de la acción educadora. ¿Cómo podremos hacer una educación eficiente si no comprendemos la naturaleza de nuestros discípulos? ¿Y cómo podremos comprenderlos si no los amamos? Pienso que, en el orden moral, sólo entendemos lo que amamos. Necesitamos esta corriente de simpatía del profesor a los alumnos para penetrar en sus intimidades psicológicas (...)"1.

Esta situación anecdótica, suerte de parusía pedagógica, narrada por un profesor que, como otros tantos de sus colegas chilenos, partió a formarse en el Teachers College de la Universidad de Columbia, puede servir como puerta de entrada a algunas de las variadas temáticas que la historiografía de la educación chilena ha debido enfrentar en los años recientes. El viaje pedagógico de Salas Marchán, inscrito en un contexto de profesionalización del campo pedagógico en Chile, forma parte de procesos de circulación de saberes educacionales en escala transnacional. Por otra parte, la impresión que provocó en el viajero chileno la actitud de la profesora frente a sus estudiantes puede ser leída en una clave que atienda a las dimensiones afectivas involucradas en la relación pedagógica. De tal modo, se le puede interpretar como un episodio que pone en juego concepciones con-

1 Max Salas Marchán, “¡Yo los ayudo!”, Revista de Educación Nacional, Año XX, n³ (mayo 1924): 157. 
trastantes acerca del talante emocional que, con sustento en los avances de la psicología educacional en proceso de consolidación en la época, se esperaba que tuvieran los adultos en la cotidianeidad de la cultura escolar.

Los dos asuntos recién señalados constituyen el núcleo de interés de este texto. Tanto las formas de internacionalización de saber pedagógico como la formulación de modelos deseables de vinculación entre profesores y alumnos (y de conocimiento más profundo de estos, a nivel de la subjetividad, basado en la psicología como un conocimiento de matriz científica) sufrieron cambios a través del siglo XX, siendo el período de la posguerra un tiempo que, por sus características de conjunto, otorgó perfiles singulares a ambos procesos. Así, mediante del análisis de documentación oficial, testimonios escritos de actores de la época y bibliografía que recogieron las posiciones de distintos intelectuales, educadores y expertos en el campo, en las siguientes páginas se busca sustentar la idea de que el proceso de instalación y legitimación de la orientación al interior de la enseñanza secundaria en Chile permite dar cuenta de fenómenos de circulación de saberes pedagógicos (ya sea en una clave global como también en lo que respecta al horizonte de políticas educacionales domésticas). Además, se sostiene que dicho proceso también es fructífero para apreciar, a modo de indicio, transformaciones (discursivas y, en menor medida, prácticas) en las dimensiones normativas respecto a lo emocional existentes en los estilos de relación entre profesores y estudiantes.

Para cumplir con los propósitos recién señalados, este artículo se divide en cuatro secciones. En una primera instancia se presentan las definiciones conceptuales a partir de las que se emprende el análisis del proceso de instalación de la orientación escolar en el sistema escolar chileno. Así, se propone comprender este fenómeno como parte de un proceso general de transnacionalización educacional. Complementariamente, en esta sección inicial se entrega una referencia sumaria a conceptos en uso en la corriente de historia de las emociones, con el propósito de emplearlos como marco de análisis de algunos aspectos del desarrollo de la orientación escolar en Chile en el período en estudio. La segunda parte del texto tiene como propósito entregar, a modo de contexto, una muy breve mirada a la orientación en algunos países que eran referentes educacionales 
de Chile previamente a la mundialización de las políticas de educación, marcada simbólicamente por el surgimiento de UNESCO en 1947. En el tercer apartado se presenta un panorama de los orígenes de la orientación en Chile, en el marco de fines del siglo XIX hasta la década de 1950. Por último, en la cuarta sección de este artículo se analizan con mayor detalle los primeros años de la instalación de la orientación en el sistema escolar chileno de posguerra, a la luz de algunas de sus dificultades prácticas y, sobre todo, de su papel de entorno para nuevas formas de vinculación emocional entre profesores y estudiantes.

\section{SOBRE TRANSNACIONALIZACIÓN EDUCACIONAL Y EMOCIONES}

El establecimiento de la orientación escolar en Chile es un fenómeno que permite apreciar la índole crecientemente global (o sea, transnacional) que adquiere la educación durante el siglo $\mathrm{XX}^{2}$. La visita de Salas Marchán a Estados Unidos, seguida por una verdadera legión de educadores chilenos en las décadas siguientes, fue un puente entre espacios de conocimiento pedagógico distintos. En una primera mirada (que definiríamos como limitada), se podría entender que procesos como el que nos convoca en estas páginas formaron parte de ciclos de "transferencia cultural". El problema de un enfoque tal es que afirma la existencia de puntos fijos de origen y de llegada de ideas o prácticas, a las que dicha noción de transferencia pretende analizar en la medida de su supuesta fidelidad a un modelo original, simplificando además los procesos a una naturaleza bilateral. Por ende, al adoptar este tipo de lectura se correría el riesgo de desconocer la complejidad de redes y el entrelazamiento (entanglement) que supone el flujo de ideas estimulado, en el marco globalizador, por los medios de transporte y los avances de la comunicación ${ }^{3}$. Así, tanto experiencias de

2 Para evitar confusiones, precisamos que, en el marco de este artículo, usamos el término "global" en su sentido intercambiable con transnacional. Global ha sido comprendido en, a lo menos, tres sentidos principales al usarlo para efectos históricos: como la historia de todo el mundo; la historia de las conexiones en escala superior al ámbito nacional y, finalmente, como una historia (con sentido normativo) interesada en el concepto de integración. La segunda acepción es la que nos parece más pertinente para nuestros fines. Sobre esta conceptualización, ver Sebastian Conrad, What is Global History? (Princeton: Princeton University Press, 2006), 6.

3 Una sistematización sobre los límites del enfoque de transferencia educacional se presenta en la primera parte del interesante artículo de Noah W. Sobe "Entanglement and Transnationalism in the History of American Education", en Rethinking the History of Education. Transnational Perspectives on Its Questions, Methods, and Knowledge, ed. Thomas S. Popkewitz (New York: Palgrave Macmillan, 2013), 95-99. 
agentes individuales de "transferencias interculturales" (como, por ejemplo, James Thompson y su misión de propaganda del método Lancaster de enseñanza mutua en Chile, entre otros países, a inicios del siglo XIX o el mismo viaje de Salas Marchán) como de orden colectivo e institucional (al estilo de la misión norteamericana que apoyó presencialmente la reforma de la enseñanza secundaria chilena a partir de 1946), perderían parte de su riqueza y complejidad al comprenderlas meramente como trayectos directos desde origen a destino. Esa merma se daría en tanto no se integren al análisis las adaptaciones, mezclas, resistencias, calcos culturales, traducciones y "desviaciones interpretativas", entre otros tantos fenómenos propios de encuentros entre ideas de culturas distintas ${ }^{4}$.

Por lo recién señalado, sostenemos que la llegada de la orientación escolar a Chile puede ser mejor comprendida si se la inscribe en el marco de los procesos de intercambio cultural de carácter transnacional. Así, rebasando los límites del enfoque de relaciones entre Estados o instituciones (aunque incluyéndolo como un elemento en ningún caso desdeñable), una mirada transnacional al tema debería tener en cuenta que modelos, referencias e ideas sobre qué se debía entender por orientación (y porqué era necesario integrarla al sistema escolar) formaron parte de objetivos compartidos por personas o grupos, no obstante, sus procedencias nacionales diferentes $^{5}$. En la escala biográfica de los contactos personales y las consecutivas influencias mutuas, por ejemplo, sería difícil no considerar experiencias como la de Irma Salas, figura clave en la reforma educacional de 1946 que instituye la orientación escolar. En su etapa de estudiante doctoral en la Universidad de Columbia, Salas residía en la Casa Internacional (albergue para estudiantes universitarios de todas partes del mundo que fue establecido por Rockefeller): una clara experiencia de transnacionalización, que involucra personas, espacios formativos e instituciones ${ }^{6}$. Del mismo

$4 \quad$ Acerca de la noción de transferencia intercultural, resulta de utilidad la consulta del estudio de Thomas Adam, Intercultural transfers and the making of the Modern World. Sources and Contexts (Londres: Palgrave Macmillan, 2012), 5.

5 Akira Iriye, Global and Transnational History: The Past, Present, and Future. (Londres: Palgrave Macmillan, 2013), 15. Para una visión evolutiva del concepto "transnacional”, ver Pierre-Yves Saunier "Transnational", en The Palgrave Dictionary of Transnational History eds. Akira Iriye y Pierre-Yves Saunier (Londres: Palgrave Macmillan, 2009), 1047-1054.

6 Emma Salas Neumann, Irma Salas. Educación e innovación en Chile (Santiago: Corporación Cultural Rector Juvenal Hernández, 2008), 20. 
modo, una mirada general a protagonistas claves de la institucionalización de la orientación escolar en Chile en el período en estudio permitiría reforzar esta idea de circuitos y contactos transnacionales, reconociendo la existencia de una red de actores que, de acuerdo a sus influencias previas, adscripciones institucionales e historias personales, formaron más bien nudos de una red flexible en vez de ladrillos de un espeso muro doctrinario. En el tejido de esa red, extendida en el tiempo, la afluencia permanente de profesoras y profesores chilenos, sobre todo a universidades norteamericanas, ha sido objeto de atención reciente por parte de Rodrigo Mayorga. Así, se constata que entre 1920 y 1953 cuarenta chilenos y chilenas cursaron estudios de posgrado en Teachers College ${ }^{7}$.

Definido nuestro problema por su dimensión transnacional, corresponde delinear, de manera muy acotada, algunos conceptos que nutren el análisis de otro aspecto que nos interesa poner en relieve al observar la entrada de la orientación a la educación chilena: la posibilidad de comprenderla desde el ángulo de las relaciones afectivas o emocionales entre los actores involucrados. Para acometer dicha tarea es necesario tener a la vista que la historia de la educación ha ido concediendo atención creciente a los aspectos afectivos implícitos en las relaciones educativas. Como se ha señalado en una revisión temática y bibliográfica reciente, el primer foco de interés de la historiografía al mirar a lo escolar desde las claves de afectos y emociones ha sido en torno a los aspectos disciplinarios y normativos, especialmente los referidos a control corporal, restricciones y "gubernamentalidad", siguiendo orientaciones teóricas provenientes de las ideas de Michel Foucault ${ }^{8}$. Por ende, ha sido frecuente que se aluda a uno de los primeros conceptos fundantes de la historia de las emociones: la noción de "emocionología". Peter Stearns y Caroline Stearns la propusieron, en

7 Rodrigo Mayorga, Actores transnacionales imperialismo cultural y prácticas discursivas en la irrupción de la sicología educacional en Chile (1930-1960). Ponencia presentada en ISCHE 2017 (Buenos Aires) Inédito: 2. Agradecemos al autor su amabilidad al facilitarnos el texto.

8 Noah W. Sobe, "Researching emotion and affect in the history of education", History of Education: Journal of the History of Education Society, v. 41, nº5 (2012): 691-692.

2909 En el caso de la historiografía de la educación chilena, el concepto ha sido usado (de manera aproximativa) en estudios como, por ejemplo, Pablo Toro Blanco, "Close to you: building tutorials relationships at the Liceo in Chile in the long 19th century", Jahrbuch für Historische Bildungsforschung, 18 (2012): 70-90. 
1985, como un "conjunto de estándares emocionales colectivos en los que agentes sociales e instituciones promueven o prohíben ciertos tipos de emociones $\mathrm{O}$, al contrario, otras les resultan indiferentes" ${ }^{\prime 10}$. Esta perspectiva surge con naturalidad si se considera la índole profundamente normalizada y estructuralmente asimétrica de las relaciones en el contexto escolar. Ahora bien, es pertinente indicar que en la enunciación de este concepto se reconoció tempranamente uno de los dilemas fundamentales que enfrentarían los investigadores en historia de las emociones: el diferencial existente entre dichas dimensiones normativas, observables en reglamentos y prescripciones, y la recepción y asimilación de tales estándares por sus destinatarios en la experiencia cotidiana. Esa brecha también forma parte de los límites que un texto como este, en función de su enfoque y fuentes, debe tener a la vista.

Para el análisis de preceptos conductuales con alusiones afectivas, enmarcados en el naciente campo de la orientación escolar, resulta necesario considerar que el dominio de lo afectivo y emocional cobra sentido históricamente a condición de entenderlo como inscrito en el ámbito de la experiencia interpersonal y, por lo tanto, de lo social. En tal sentido, las emociones aparecen permanentemente referidas a marcos que las interpretan (ya sea con base en normas morales, religiosas o de otra base prescriptiva). Por ende, resultan ser instancias que remiten, con mayor o menor transparencia, a relaciones de poder. Estas observaciones, que podrían resultar obvias o redundantes, son necesarias en la medida que se requiere mirar a afectos y emociones en una clave que sobrepase una comprensión meramente neurobiológica, que los entiende principalmente como circuitos de estímulo-respuesta aposados en los arcanos del sistema nervioso. En consecuencia, afectos y emociones están necesariamente enunciados en gestos corporales o materiales, lo que supone la asociación de expresiones faciales con emociones específicas. Ello constituye una "carta de navegación” para su legibilidad y manejo (en nuestro caso, por ejemplo, para el reconocimiento e interpretación de estados afectivos en los estudiantes,

10 Peter Stearns y Caroline Stearns, "Emotionology: Clarifying the History of Emotions and Emotional Standards", The American Historical Review, v. 90, n4 (1985): 813. La traducción es nuestra. 
a los que la orientación escolar consideraba como parte de su ámbito de acción $)^{11}$.

\section{Antecedentes de la llegada de la orientación escolar a Chi- LE: Sus inicios y Desarrollo antes de la Segunda Guerra Mun- DIAL}

La convicción que Salas Marchán tenía acerca de la necesidad de establecer esa "corriente de simpatía del profesor a los alumnos para penetrar en sus intimidades pedagógicas" no tenía, a fines de 1918, la posibilidad de expresarse en un espacio específico al interior del tablero de las disciplinas escolares reconocidas institucionalmente en Chile. Si bien la psicología educacional estaba dando ya sus primeros pasos en el país (como lo demuestran su enseñanza en el Instituto Pedagógico hacia 1895 y el establecimiento del primer laboratorio de psicología experimental, a cargo de Guillermo Mann, en 1908), ello no implicaba que existiera una proyección de sus investigaciones que estuviera integrada sistemáticamente al currículo escolar ${ }^{12}$. En la primera parte del siglo XX, las inquietudes de los docentes e intelectuales educacionales criollos respecto a la necesidad de establecer nuevos patrones de relación con los estudiantes, privilegiando los aportes de la psicología por sobre la mera herencia de preceptos morales o religiosos, no tuvieron espacio de expresión en los términos que la moderna historia de las disciplinas escolares identifica como necesarios para constituirse como tales. Así, atendiendo a las referencias que Antonio Viñao Frago hace a autores como Ivor Goodson o André Chervel, se pueden detectar las carencias, a inicios del siglo XX, del ámbito de lo que sería la futura orientación escolar: no disponía de un código disciplinar reconocido, o sea, "un cuerpo de contenidos (saberes, conocimientos, destrezas, técnicas, habilidades), un discurso o argumentos sobre el valor formativo y la utilidad de los mismos, y unas prác-

11 Las prevenciones señaladas en el párrafo están planteadas por Jan Plamper, en su instructivo libro The history of emotions. An introduction. (Oxford: Oxford University Press, 2015), 39.

12 La referencia a los albores de la enseñanza de la psicología en la formación de los profesores de enseñanza secundaria, mediante la cátedra del profesor alemán Jorge Schneider, la presenta Gonzalo Salas, "Pasado y Presente de la Psicología Científica en Chile: Profesionalización, instituciones y divulgación científica”, en Historias de la Psicología en América del Sur. Diálogos y perspectivas, ed. Gonzalo Salas (La Serena: Nueva Mirada Ediciones, 2014), 102. 
ticas profesionales"13. Sus contenidos estaban todavía por definirse, como se verá, en un movimiento pendular entre la preparación para el mundo del trabajo y la atención psicológica al desarrollo formativo de los estudiantes. Su capacidad de validarse como necesaria estaba lejos de tener los rendimientos suficientes como para justificar un espacio, aunque fuera mínimo, en la grilla de aprendizajes de la escuela y, sobre todo, todavía no se apreciaba en el horizonte un cuerpo de intérpretes que fuera capaz de traducir en prácticas sus focos de interés.

Carente de una definición disciplinar clara, la orientación escolar arribó a las costas de la educación chilena como parte de un movimiento de circulación transnacional de ideas pedagógicas. En este sentido, si se sigue la argumentación de autores como Ramírez y Meyer, podría entenderse que su proceso de adopción formaría parte de los primeros episodios de una tendencia hacia el "isomorfismo educacional" propio del siglo XX, en la medida que elementos curriculares que representan valores hegemónicos con eficacia global (o en camino de serlo, mediante la inducción generada desde los focos de irradiación, sean estos académicos o agencias multilaterales), suelen ser integrados en los currículos nacionales ${ }^{14}$. Así, valores o propósitos educacionales en expansión como, por ejemplo, el autodominio, la búsqueda del equilibrio emocional o la adaptación al horizonte laboral, tendrían su expresión en el campo de acción de la orientación escolar.

Es importante relevar la necesidad de evitar caer en anacronismos o esencialismos al mirar los orígenes de la orientación escolar, tanto en sus primeras formulaciones en los países que le servían como referencia admirativa como también en Chile. Una consideración indispensable es que, por su índole transnacional, lo que se identificaría como orientación educacional, escolar o vocacional en Chile en la posguerra resultó ser final-

13 Antonio Viñao Frago, "La historia de las disciplinas escolares", Historia de la Educación, n²5 (2006): 267.

14 Francisco O. Ramírez y John W. Meyer, "Los currículos nacionales: modelos mundiales y legados históricos nacionales", en Internacionalización. Políticas educativas y reflexión pedagógica en un medio global, compiladores Marcelo Caruso y Heinz-Elmar Tenorth (Buenos Aires: Granica, 2011), 116. 
mente el producto contingente, con elementos de hibridez, de un campo de conocimiento muy amplio. Así, compartiendo la preocupación por el bienestar personal de niños y jóvenes, por una parte, y la inquietud por su adaptación lo más funcional posible al sistema político y al mercado laboral, la orientación tuvo énfasis bastante diversos de acuerdo a cada tradición nacional, del mismo modo que las denominaciones se traslaparon y confundieron sus bordes en más de una ocasión. Es el caso de la idea de counselling, noción que inicialmente, en los Estados Unidos, tenía que ver con un acompañamiento de un adulto a un estudiante, fundamentalmente para analizar su futuro laboral o académico, y que cambió de sentido en ese mismo país conforme se instaló, desde la posguerra, una vertiente de orientación escolar con un componente más centrado en el acompañamiento psicológico.

Hacia inicios del siglo XX en Estados Unidos florecieron diversas muestras de interés por auxiliar a los estudiantes tanto en su autoconocimiento y autogestión como en su preparación para el futuro desempeño exitoso en el mundo del trabajo. Respecto a lo primero, se identifica a Jesse B. Davis, autor de Vocational and Moral Guidance (1914), como uno de los pioneros de la asociación entre educación escolar y orientación comprendida como proceso de exploración y cultivo de la personalidad y el carácter de los estudiantes. En cuanto al foco de la orientación como apresto para la elección del futuro laboral, se suele considerar como un punto crucial la publicación, en 1909, de Choosing a Vocation de Frank Parsons. El hecho de que su propuesta articulara un método de etapas sucesivas para la elección vocacional (autoconocimiento, acopio de información y ajuste al campo de desempeño laboral) le concedió a la orientación vocacional un estatuto reconocido en el medio educacional norteamericano ${ }^{15}$.

Los antecedentes recién señalados formaban parte del acervo de discusiones pedagógicas que se daban en los Estados Unidos en el primer cuarto del siglo XX, época en la que ya se había establecido un flujo regular de viajeros pedagógicos chilenos que peregrinaban a tierras norteamericanas,

15 Lawrence K. Jones, “Frank Parsons' contribution to career counseling”, Journal of Career Development, v. 20, nº (1994): 294. 
muchos de los cuales tendrían incidencia en las políticas educacionales en Chile en las décadas siguientes. Es importante considerar que las propuestas de las dos ramas principales de la orientación escolar tenían puntos en común, los que es plausible imaginar que resultaban de sumo interés para los educadores nacionales: la búsqueda por establecer afirmaciones de base científica (o sea, psicológica), con apoyo en la experimentación y el uso creciente de mecanismos seriales de reporte (encuestas, cuestionarios); la adhesión al "giro paidológico" en curso en la época, lo que implicaba albergarse teóricamente bajo el manto genérico de la Escuela Nueva; y, por último, la preocupación por resaltar las interfaces necesarias entre educación, estabilidad política y crecimiento económico ${ }^{16}$. No resulta extraño, pues, que la orientación escolar norteamericana, a la luz de estas características, comenzara a ser tomada en cuenta como un modelo a adoptar y adaptar al caso chileno.

Cabe agregar como último elemento de esta breve caracterización transnacional del campo de la orientación que el sistema escolar chileno había tenido históricamente una estrecha relación de admiración respecto a la educación francesa, lo que se había expresado en buena parte de su estructura. De hecho, la organización de la enseñanza secundaria chilena, con el sistema del liceo, había sido una temprana importación desde el referente francés y la influencia de la pedagogía gala se había extendido, casi sin contrapeso, durante la mayor parte del siglo XIX en Chile. El sistema educacional francés acuñó un concepto de la orientación que definió como un propósito privilegiado la preparación de los estudiantes para sus desempeños futuros, gestando un sistema de acreditación de aptitudes laborales y de apoyo. Así, desde 1922 se fueron creando los Centros de Orientación Profesional, fuera del sistema escolar propiamente tal, en los que se integraba un análisis de los estudiantes a partir de sus dimensiones escolares, sociales y de potencialidad laboral, con un claro basamento en un enfoque

16 Una muestra de este interés de los profesores chilenos por darle base científica (esto es, psicológica) y médica a la pedagogía se encuentra presente en el movimiento funcionalista, surgido en la década de 1920. Una buena caracterización de esta tendencia se encuentra en el texto de Iván Núñez Prieto, "Biología y educación: Los reformadores funcionalistas. Chile, 1931-1948", Cuadernos Chilenos de Historia de la Educación, nº1 (2013): 65-86. 
psicológico y experimental ${ }^{17}$. No obstante, pese a la tradicional ligazón entre la educación francesa y la chilena, este modelo no tuvo la influencia que sí logró el norteamericano, como se ha de notar en los siguientes párrafos.

\section{Antecedentes nacionales de la llegada de la ORIENTACión ES- COLAR A LOS LICEOS}

A raíz de la mención reciente al sentido fundante y estructural de la influencia francesa en la educación chilena, antes de apreciar los primeros derroteros de la orientación escolar en Chile se requiere explicitar una condicionante estructural para el desarrollo de la orientación escolar como espacio disciplinar dentro de la educación chilena durante buena parte del siglo XX: el sistema educacional chileno estaba dividido en dos caudales que tenían escasa comunicación entre $\mathrm{si}^{18}$. Esa bifurcación no era un mero calco cultural de la educación y sociedad francesa, pero sí es importante considerar que ambos sistemas nacionales de educación se desarrollaron durante el siglo XIX a partir de un dualismo marcado entre instrucción popular primaria y enseñanza secundaria humanística-literaria y científica, en función de decisiones políticas de priorizar a la segunda para fortalecer la formación de un grupo dirigente capacitado para manejar la Nación ${ }^{19}$. De hecho, las instituciones representativas de cada orientación fueron tomadas por Chile a partir del modelo francés: la creación temprana del Instituto Nacional (1813) y el establecimiento de un sistema de capacitación docente mediante la Escuela Normal de Preceptores (1842). De tal suerte, cuando la orientación escolar comienza sus primeros pasos, el legado del siglo XIX establecía que la educación secundaria tradicionalmente había estado dirigida hacia los sectores medios y altos $\mathrm{y}$, a mediados del siglo $\mathrm{XX}$, todavía se argumentaba que su propósito (o al menos uno de los

17 Marcel Turbiaux, “J.-M. Lahy (1872-1943) et l'orientation professionnelle”, Bulletin de psychologie, 482/2 (2006): 220-222.

18 Iván Núñez, entre otros autores, señala esta fisura como basada en criterios de clase. Iván Núñez, Desarrollo de la Educación Chilena hasta 1973 (Santiago: PIIE, 1982), 6.

19 Esta diferencia de valoración llevó a que, en 1862, la inversión por un alumno en secundaria fuera equivalente a la de doce estudiantes de primaria, diferencia que se fue atenuando, aunque no desapareció, a través del siglo XIX. Al respecto, ver el completo estudio que entrega la obra de Sol Serrano, Macarena Ponce de León, y Francisca Rengifo (editoras), Historia de la Educación en Chile (18102010). Tomo I. Aprender a leer y escribir (1810-1880). Santiago: Taurus, 2012), 243-244. 
principales) era la formación de una elite dirigente a partir de la idea de meritocracia. Por otra parte, la instrucción primaria, históricamente concebida como una herramienta de normalización de los sectores populares, tenía un objetivo principalmente nacionalizador y (más declarativamente que en los hechos) de preparación al rol de mano de obra que los estudiantes deberían desempeñar luego de asistir a ella. Naturalmente, la falta de correlación y conexión entre ambos sistemas habría de tener implicancias mayores en el desarrollo de la orientación escolar.

Como consecuencia del divorcio entre instrucción primaria y educación secundaria se produjeron dos comprensiones distintas acerca del sentido y razón de la orientación escolar en el escenario de la primera mitad del siglo XX. En un primer caso, fue entendida básicamente como una forma de apresto para la elección vocacional. Esta tarea se ejecutó mediante el ejercicio de trabajos manuales (como era el supuesto de funcionamiento de la enseñanza vocacional surgida en la década de 1920) y también a través del acopio de información, mediante encuestas, sobre expectativas laborales de los sectores populares asistentes a las escuelas primarias y la entrega de información a los alumnos sobre el mundo del trabajo. En contraste, para la educación secundaria el sentido de la orientación incluyó también la preocupación por el horizonte laboral futuro de los estudiantes, en atento seguimiento a la evolución de los campos de desempeño profesional, pero agregó un interés sostenido por conocer la personalidad o carácter de los estudiantes mediante herramientas estandarizadas (cuestionarios, entrevistas) y poder incidir sobre ella, en términos de estimular destrezas y actitudes determinadas. En este segundo caso, este enfoque logró materializarse solamente de forma acotada, durante las décadas de 1930 y 1940, en el Liceo Manuel de Salas, uno de los bastiones de la experimentación pedagógica de la educación secundaria en Chile. El carácter de experimental se refiere a que implementó (de manera intensa pero limitada solamente a su entorno inmediato) muchas de las instancias educativas que luego se harían extensivas al conjunto de los liceos del país: clases de orientación, consejo de curso y gobierno estudiantil, servicio de psicología educacional, coeducación, entre otras.

La experiencia del Liceo Manuel de Salas se hallaba inscrita en el marco de 
una intensa reforma educacional (que tuvo un episodio cimero en Chile durante la dictadura de Carlos Ibáñez del Campo, 1927-1931). Así, la legislación que normaba la enseñanza secundaria recogió indicios de lo que caracterizaría en los años siguientes a la orientación escolar: el Decreto 2693, de fecha 20 de junio de 1928, establecía que el profesor debería "mantener una relación permanente y de confianza familiar con sus alumnos; servirles de consejeros en sus dudas y vacilaciones y ayudarlos a conseguir el máximo aprovechamiento de su permanencia en el colegio" ${ }^{20}$. De acuerdo a una de las protagonistas de la profesionalización de la orientación escolar en Chile, este tipo de disposiciones reflejaba los primeros avances de este campo que, según su juicio, llegaría a constituir "uno de los soportes internos de la escuela en la democratización de la educación"21.

Siguiendo la premisa de que la posibilidad de la orientación de erigirse en campo disciplinar dependía de una articulación de un campo de conocimiento, prácticas y actores legitimados que se coronara en presencia curricular, es interesante notar que en los años siguientes se encuentran testimonios (al margen del espacio experimental ya señalado) que dan cuenta de iniciativas aisladas, de escasa formalización o de alcance meramente local. A título de ejemplo, baste considerar que en la formación de los profesores y en la creciente investigación pedagógica que se daba en la época había interés explícito por los problemas de orientación vocacional y se echaba mano a toda herramienta que pudiera ser de utilidad, pese a la carencia de medios institucionales. En julio de 1928 el rector del Liceo de Concepción recibía una carta de la Escuela de Educación de la Universidad de Concepción. En ella se le informaba que el Departamento de Orientación Profesional de ese centro de estudios superiores había inaugurado recientemente un Consultorio Psico-Pedagógico y se invitaba a que los profesores del Liceo llevaran a sus estudiantes que tuvieran dudas vocacionales para recibir el auxilio de los especialistas ${ }^{22}$. Sin embargo, a pesar de lo virtuosa que podía resultar la vinculación entre investigación

20 Decreto n²693, Reglamento General de la Educación Secundaria, artículo 78.

21 Emma Salas Neumann, Apuntes sobre educación y algunos retazos (Santiago: sin dato editorial, 2010), 38.

22 “Carta al rector del Liceo de Concepción”. 25 de julio de 1928. Archivo del Liceo Enrique Molina Garmendia (ALEMG), s/n/f. 
universitaria y educación secundaria, este tipo de instancias distaban de convertirse en políticas educacionales consistentes.

Otro caso que da testimonio del interés de los establecimientos secundarios por allegar conocimiento con base psicológica sobre sus estudiantes es el que se presentó en el Liceo de Niñas de La Serena, a fines de 1940. Precediendo en algunos años a la existencia formal de la orientación escolar en la enseñanza secundaria, se producía una colaboración entre el Liceo y la Escuela de Servicio Social mediante la cual se implementó un instrumento de observación de las estudiantes del VI año de Humanidades. A las alumnas se les calificó respecto a varias categorías junto con dimensiones algo más convencionales, relativas a las dimensiones cognitivas y habilidades escolares, figuraban otras que llaman la atención: dominio de sí misma; espíritu de perfeccionamiento; condiciones morales; tacto y discreción ${ }^{23}$. El interés de las profesoras del Liceo por las dimensiones afectivas y psicológicas de sus estudiantes encontraba así un instrumento ocasional para poder caracterizarlas. No obstante, también constituyó una experiencia específica, que no formaba parte de las normativas vigentes y que, por lo mismo, no tuvo proyección en el tiempo, de acuerdo a lo que muestran las fuentes.

\section{LA ORIENTACión ESCOLAR LLEGA A LOS LICEOS}

Quien ingresara al Salón de Exposiciones de la Universidad de Chile, en la primera semana de diciembre de 1946, se encontraría con una muestra del recientemente creado Instituto de Guía y Orientación Profesional. A través de una serie de cuadros y láminas acompañados de sus textos respectivos, este organismo, dependiente de la Dirección General de Educación Primaria, presentaba al público un panorama respecto a qué era la orientación escolar vocacional y por qué debía ser considerada como parte indispensable de "una escuela primaria mejor". Entre las consecuencias virtuosas de una correcta orientación se destacaba que:

23 “Consejo Extraordinario. 19 de diciembre de 1940". Volumen Actas del Consejo 1939-1946. Archivo del Liceo de Niñas Gabriela Mistral de La Serena (ALNGM). En la fuente no se identifica con claridad a qué Escuela de Servicio Social se hace referencia. 
"en el orden social, una mejor organización de la familia, aumento en calidad y en cantidad de los rendimientos, disminución de los riesgos por accidente, disminución de los inadaptados, optimismo y fe colectivos, pueden ser fáciles frutos de la orientación profesional, todo lo cual repercute a favor del individuo.

Las expresiones de este provecho social se traducen en disminución de la mortalidad, vagancia, mendicidad y abandono de la infancia; en paz de los hogares; en aumento de la economía nacional; en disminución de los inadaptados, de los criminales, de los enfermos y lisiados, de los alienados y neuróticos"24.

Estas consideraciones, con foco en una lectura que podría catalogarse como de "profilaxis social", no agotaban, ciertamente, el sentido de lo que se esperaba de la orientación vocacional dirigida a las niñas y niños asistentes a las escuelas primarias en Chile. Sin embargo, nos parece interesante rescatarlas en una doble función respecto a nuestros intereses. En primer lugar, debido a que resaltaban el contenido prioritario que se le siguió asignando en la posguerra a la orientación en la educación primaria: inserción exitosa en un orden laboral que permitiría sortear todas las lacras recién descritas. Además de ello, identificaban tanto taras (la mayoría) como condiciones beneficiosas (optimismo, por ejemplo) sustentadas en situaciones conductuales, de matriz psicológica, posibles de reducir a estándares en buena parte de los casos. Esta enumeración de cuadros clínicos o estados emocionales parecía mantener una filiación estrecha con buena parte de las preocupaciones y objetos de la orientación de la escuela primaria del período anterior.

Algunas notas contrastantes con lo recién señalado pueden ser apreciadas, en cambio, cuando se dirige la mirada hacia la orientación escolar en el ámbito de la enseñanza secundaria en el escenario de la posguerra, dado que parece haber ofrecido un suelo más propicio para promover cambios

24 Ministerio de Educación. Dirección General de Educación primaria. Instituto de Guía y Orientación Profesional, Exposición Técnica de Orientación Profesional (Guía para el visitante). "Por una Escuela Primaria Mejor" (Santiago: sin datos de editorial, 1946), 26. 
de mayor profundidad cualitativa, a la luz de los avances ya señalados en la experimentación llevada a cabo por más de una década en el Liceo Manuel de Salas (que siempre fueron entendidos como un preludio de políticas que deberían generalizarse al conjunto de los liceos de Chile).

En segundo lugar, muy estrechamente ligado al primer factor, la capacidad logística de implementar transformaciones limitadas podría ser interpretada como una condición favorable, sobre todo en el nuevo escenario de las relaciones educativas transnacionales que caracterizaría a la posguerra. Con esto nos referimos a que, tanto desde el lado de la oferta (organismos como la Fundación Interamericana de Educación) como desde el de la demanda (en este caso, el Ministerio de Educación de Chile), una reforma educacional aplicada sobre un espacio acotado parecía presentar mayor viabilidad y posibilidades de demostrar frutos en tiempos delimitados y políticamente redituables para un gobierno. Esa parecía ser la apreciación de los organismos técnicos estadounidenses al promover para la enseñanza secundaria en Chile un programa que, mediante reformas graduales y aproximaciones sucesivas, pudiera garantizar una "evolución racional, progresiva y eficaz" y servir como una suerte de experiencia piloto a ser implementada posteriormente en otros países de América Latina ${ }^{25}$.

Así, en sintonía con los énfasis educacionales que guiaban a los gobiernos de coalición con hegemonía del partido radical, en los primeros años de la posguerra Chile estableció lazos sólidos con la Fundación Interamericana de Educación, proyección continental de los intereses norteamericanos de construir alianzas en el nuevo escenario hemisférico. Se suscribió en marzo de 1945 un convenio para implementar una Reforma Gradual de la Enseñanza Secundaria, que involucraba la asesoría de profesores norteamericanos a sus pares chilenos en áreas detectadas como sensibles para la transformación de los liceos, los que venían siendo objeto durante décadas de críticas por su tradicionalismo y falta de adecuación a los cambios culturales y sociales. Entre dichas áreas se encontraba la orientación. Por ello, en septiembre de 1945 se creó el Departamento de Orientación y Educación Vocacional en el Ministerio de Educación.

25 La renovación del Liceo Chileno (Washington: Publicaciones de la Unión Panamericana, 1945): Introducción. 
De acuerdo a las orientaciones teóricas que se indicaban al inicio de estas páginas, para poder alcanzar un perfil disciplinario se requería que la orientación en la enseñanza secundaria lograra satisfacer algunos criterios: establecer reconocimiento a sus saberes y técnicas; generar consenso respecto a su valor formativo y disponer de un cuerpo de profesionales que ejecutara regularmente sus prácticas. Todas esas tareas resultaban complejas, si se considera que parte importante del legado de las décadas anteriores había permanecido aherrojado en círculos muy pequeños de docentes y estudiantes. Por ende, no es de extrañar que la propaganda oficial respecto a la orientación como componente del Plan de Reforma Gradual de la Enseñanza Secundaria apostara a enfatizar su novedad y pretendiera marcar distancias respecto a las características del muy criticado liceo público tradicional. Acerca de lo primero, para resaltar la novedad del Plan un documento adaptado de una fuente norteamericana integraba a la reforma en curso en un proceso en que "la investigación científica ha descubierto y compilado más conocimientos acerca del individuo y de su crecimiento físico, mental y emocional en los últimos cincuenta años que lo que se había logrado en todo el tiempo precedente" ${ }^{26}$. Respecto a lo segundo, se puede encontrar testimonio de ello en un boletín publicado en 1946 que, con el propósito de socializar los ideales del reformismo pedagógico de la posguerra entre el profesorado nacional, presentaba gráficamente una contraposición de múltiples dimensiones entre el liceo tradicional y el liceo renovado. En lo que respecta al campo de la orientación escolar, se sostenía que mientras en el primero "el alumno carece de guía” en el liceo renovado "el servicio de Orientación lo ayuda a solucionar sus dificultades escolares y, muy principalmente, los problemas de su vocación". Complementariamente, frente a un liceo tradicional que miraba al estudiante como "inteligencia pura", se afirmaba que "la doctrina progresista lo trata como una persona con sentimientos y aspiraciones propias" 27.

Establecer un quiebre de la disciplina con el pasado y resaltar su pertinencia eran tareas que los escasos especialistas en orientación secundaria

26 Ministerio de Educación Pública. Comisión de Renovación Gradual de la Educación Secundaria. Disciplina (Santiago: Imprenta y Litografía Stanley, 1947), 14.

27 Boletín Renovación, año I, nº1 (1946): Introducción. 
requerían cumplir cabalmente. En los esfuerzos conducentes a ello es posible detectar interesantes trazos de atención a la formación de la personalidad de los estudiantes, a su proceso de individuación y subjetivación. Desde ya, cabe señalar que la atención a la singularidad buscó ser conducida mediante diversos cauces, uno de los cuales fue la incorporación de cursos electivos en el currículo secundario y el establecimiento de una orientación vocacional sistemática ${ }^{28}$. Paralelamente, en busca de cumplir con el baremo de su utilidad, la orientación fue promovida como una necesidad formativa. Enrique Salas, uno de los principales protagonistas del proceso de inserción de la orientación en la escuela secundaria durante esos años, sostenía en 1946 que "del mismo modo que cuando emprendemos un viaje necesitamos saber lo que llevamos en nuestra valija, nos es también indispensable conocer el bagaje de potencialidades y limitaciones de nuestra personalidad para hacer de nuestro paso por la vida una experiencia noble y feliz"29.

De acuerdo a la estrategia de la Reforma Gradual de la Enseñanza Secundaria solamente algunos liceos incorporarían servicios de orientación escolar, así como el resto de las innovaciones contempladas en el Plan. En su formulación inicial, este se ejecutaría con la asesoría norteamericana durante un plazo de cinco años para luego ser sometido a evaluación. No obstante, fue la propia Fundación Interamericana de Educación la que decidió dar de baja la cooperación con Chile antes de que se cumpliera el plazo establecido. Pese a ello, la Reforma se mantuvo y definió como horizonte el año 1953 para que se llevara a cabo la masificación al sistema de los cambios establecidos en los liceos reformados. Estas singularidades del proceso generaron que, en sus primeros años, el grado de arraigo de la orientación escolar en la enseñanza secundaria fuera bastante volátil. Sin embargo, más allá del problema normativo, un punto que conspiró especialmente contra el asentamiento de la orientación en los liceos fue la inexistencia de un cuerpo de intérpretes y técnicos calificados que la pudiera implementar, incluso en la escala experimental en que fue instalada en el período 1946-1953.

28 Armando Samper, Technical cooperation in Latin America. A Case Study of Cooperation in Secondary Education in Chile (Washington: National Planning Association, 1957), 21.

29 Enrique Salas, "La Orientación Educacional y Vocacional en el Nuevo Liceo", Revista de Educación (1947): 69. 
La escasez o inexistencia de orientadores fue, pues, una barrera para la expansión de la orientación escolar en el marco reformista. No existía en el sistema universitario una especialidad formativa docente semejante, de modo tal que los cupos para llenar los cargos de orientadores en los liceos renovados a partir de 1946 tuvieron que ser asignados confiando en una ecuación que, voluntarismo y transacciones e influencias personales mediante, combinaba motivación de parte de los profesores interesados y criterios personales de propuesta y selección de parte de los cuadros directivos de los liceos y de la Dirección general de Enseñanza Secundaria. Las primeras cohortes de orientadores tuvieron cursos intensivos de formación con los asesores norteamericanos y profesores chilenos durante el verano de 1946 para luego intentar insertarse en sus establecimientos y cumplir con el nuevo rol que se les exigía, el que debía ser deseablemente compatible con sus tareas docentes previas. Así, profesores de inglés o de historia devinieron en orientadores escolares mediante una acelerada inducción que no estuvo exenta de dificultades prácticas. En el Decreto n¹336, de 17 de febrero de 1949, se establecía un baremo bastante alcanzable para quienes quisieran desempeñar ese rol: “estar en posesión del título de Profesor de Estado y haber ejercido la docencia en un establecimiento de Educación Secundaria por lo menos durante cinco años. Se estimarán como antecedentes meritorios haber frecuentado cursos de carácter pedagógico y realizado investigaciones sobre Psicología y Ciencias de la educación" 30 .

En el contexto de un acuerdo mayoritario del gremio docente respecto a la pertinencia de guiar de mejor forma a los estudiantes en su elección vocacional y en sus desempeños al interior de los liceos, la legitimación de la necesidad de la orientación no parece haber sido su mayor desafío. Sin embargo, era necesario establecer con claridad los perfiles del nuevo campo al interior del currículo escolar. Enrique Salas, director del Departamento de Orientación Educacional y Vocacional, en comunicación a los rectores de los liceos de Chile, los sintetizaba de la siguiente manera:

30 "Reglamento del Servicio de Orientación Vocacional de los Liceos", 17 de febrero de 1949, Archivo del Liceo de Hombres de Puerto Montt (ALPM), volumen Circulares 1948-49, f.159. 
"debemos partir de la base de que la Orientación Educacional y Vocacional, aun cuando para su trabajo debe recurrir a la clase sistemática, no es una asignatura más. Es un servicio especial que por medio del empleo de ciertas técnicas y de la colaboración de diversos agentes, procura ayudar a los alumnos en la solución de sus problemas, a conocerse mejor a sí mismos y, sobre todo, a formularse metas nobles y significativas. Este trabajo de orientación u consejo no puede realizarse sin un conocimiento científico del niño ${ }^{31}$.

El programa así trazado introducía un conjunto de exigencias nuevas a los establecimientos, lo que conllevó algunas tensiones ocasionales sobre la interpretación del estatus del Consejero Estudiantil. De hecho, el término que lo designaba era usado de manera intercambiable con el de Orientador Escolar. El cargo siguió experimentando cambios de nomenclatura. Así, en las normativas de 1953 se les llamaba Consejeros Vocacionales, lo que parecía restringir su labor a "tareas parciales de exploración vocacional", de acuerdo a un crítico de este enfoque ${ }^{32}$. Por ello, en 1958 se cambió la denominación a Orientadores Educacionales, que debían preocuparse de un amplio espectro de asuntos "tales como el aspecto educacional, vocacional, personal, familiar, recreativo, cívico y social, ético, estético, físico y de la salud y otros campos afines" 33 .

En tanto componente de una reforma más compleja, la entrada de los orientadores a los liceos estuvo repleta de hiatos legislativos, administrativos y prácticos. Así, por ejemplo, en el inicio del año escolar de 1947, el rector del Liceo de Concepción se encontraba perplejo ante el hecho de que, el día 21 de marzo, había recibido notificación de la creación del Servicio de Orientación en su establecimiento pero, ya pasado un mes desde esa noticia, ignoraba quién iba a hacerse cargo de él. Señalaba que, por la

31 "Comunicación de Enrique Salas Silva, Jefe del Departamento de Orientación Educacional y Vocacional al Rector del Liceo de Concepción”, 21 de marzo de 1947, ALEMG, volumen Correspondencia recibida 1947, f.1.

32 Oscar Fabres Villarroel, "La orientación educacional de la escuela secundaria fiscal”, Museo Pedagógico de Chile (1991): 4.

33 Oscar Fabres Villarroel, "La orientación educacional”, 4. 
misma razón, "nada he dispuesto respecto al local donde debería funcionar su oficina" 34 . Finalmente, se recibió el nombramiento de Raúl Parada Ritchie, profesor de Inglés, como Consejero Educacional y Vocacional del plantel. Transcurridos ya algunos años, la precariedad administrativa se mantenía. Así, Víctor Romero, rector del Liceo de Hombres de Puerto Montt, respondía a las consultas del Jefe del Servicio de Orientación Educacional y Vocacional indicando que todavía su establecimiento no disponía de un orientador y que las tareas correspondientes las llevaban a cabo los profesores jefes. Concluía su informe solicitando elementos mínimos con los cuales apoyar la tarea de estos: modelos de fichas escolares, circulares sobre normativas y funciones de los orientadores, así como materiales para llevar adelante los Consejos de Curso ${ }^{35}$.

En sus primeros años de existencia como parte del nuevo currículo de la educación secundaria, la orientación en los demás liceos de Chile debería enfrentarse a las dificultades propias de un espacio incierto: un cargo que debía insertarse en el organigrama de cada establecimiento secundario y concitar el consenso y colaboración activa de sus colegas profesores. Acerca de lo primero, no siempre existía claridad en las autoridades locales respecto a la índole de la nueva figura del consejero u orientador. El mismo rector del Liceo de Puerto Montt, informando al Director General de Educación Secundaria, sobre el estado general de su establecimiento, identificaba a la orientación escolar como un "servicio especial" del cual, como recién se señaló, su liceo carecía ${ }^{36}$. Respecto a lo segundo, es interesante notar que los protagonistas de la orientación escolar debieron construir una imagen de su labor que los legitimara ante el resto de los actores. Transcurrido no mucho tiempo de la creación de los Consejeros Educacionales y Vocacionales, uno de ellos hacía una evaluación de su funcionamiento en términos sumamente laudatorios caracterizándolos como "una nueva fuerza que, humana y científicamente, empieza a lograr

34 "Referente al Servicio de Orientación Educacional y Vocacional", 16 de abril de 1947, ALEMG, volumen Correspondencia despachada 1947, f.96.

30635 "Labor del Comité de Profesores Jefes", 13 de junio de 1953, ALPM, volumen Correspondencia Enviada 1953, f.121.

36 "Datos solicitados por circular $n^{\circ} 70$, de 25 de septiembre de 1953", 5 de octubre de 1953, ALPM, volumen Correspondencia Enviada 1953, f.225. 
una renovada interpretación de los valores actuales y potenciales del joven, encauzando su acción dentro de moldes más de acuerdo con su propia realidad integral". Ciertamente desde el interés corporativo, pero también reflejando la novedad que involucraba la orientación escolar en el liceo, agregaba que "la sensibilidad delicada del adolescente encuentra ahora una agencia a la que tiene oportunidad de recurrir con confianza y espontaneidad máximas" 37 .

Resulta interesante notar que la alusión a los dominios de lo afectivo como un componente central de la tarea de los orientadores fue uno de los elementos con los que se construyó su discurso de legitimación. El despliegue de argumentaciones acerca del talante emocional que se esperaba de ellos puede ser analizado desde el campo de la historia de las emociones. Así, la demanda a los orientadores tenía, a lo menos, un doble vínculo con aspectos afectivos: en primera instancia, porque se esperaba que fueran capaces de inducir, mediante una combinación de técnicas de manejo de diálogo, un entorno emocional favorable a la apertura de los estudiantes, de modo de acceder a sus inquietudes más profundas. En una suerte de desiderátum del orientador, adaptado de un texto norteamericano (Guidance in Secondary Schools, de Shirley Hamrin y Clifford Erickson), se planteaba que:

"Debo crear una atmósfera de confianza de manera que el alumno sienta que habla con un amigo que no lo traicionará, sino que le ayudará a resolver sus problemas (...) Debo ser sincero y comprensivo. Por lo tanto, debo interesarme verdaderamente por los problemas y las dificultades de otras personas. El compartir el punto de vista de otro, comprendiendo sus esperanzas, sus temores, sus éxitos y fracasos, es el fundamento mismo de la orientación" 38 .

37 Mariano Rocabado Muñoz, "El Servicio de Orientación en los liceos chilenos (Comentario sobre las actividades de Orientación Educacional y Vocacional realizadas por los Consejeros durante I947”, en La orientación vocacional en la Enseñanza Secundaria (Santiago: Ministerio de Educación, 1948), 23.

38 "Ideario del Profesor Orientador". Boletín del Liceo Experimental Manuel de Salas. Año I, n 2 (1944): 55. 
La generación de un entorno amigable para el consejo educacional y vocacional debería idealmente redundar en que la oficina del orientador fuera para los estudiantes una suerte de "refugio emocional", aludiendo a la categoría desarrollada por William Reddy, quien los ha definido como instancias de encuentro que escapan a los valores emocionales hegemónicos que pueden implicar sufrimiento emocional ${ }^{39}$. Así, en el ideario recién aludido y bajo condiciones ideales, la relación entre consejero y estudiante tendría como producto una situación psicológicamente liberadora para este. Sin embargo, la segunda arista de las demandas de orden emocional supuesta en la formulación de los deberes del orientador involucraba además una operación más compleja, que suponía una transformación personal, en la medida que contemplaba un talante emocional específico (interesarse verdaderamente por el otro y compartir sus puntos de vista). De tal modo, en 1946, en un texto escrito especialmente para la reforma gradual en curso, se resumía este mandato para cada futuro consejero sosteniendo que: "debe saber y ser capaz de interesarse auténticamente por todo lo que dice el alumno" 40 .

Aparece en esta exigencia ya esbozado uno de los componentes de lo que sería en las décadas siguientes una constante en la construcción de perfiles profesionales, sobre todo en las áreas expuestas a más altas tasas de interacción personal: la capacidad de desplegar una emoción en el entorno laboral pero además de ello efectivamente sentirla. Esta noción, bajo el rótulo de "trabajo emocional", fue planteada por la socióloga Arly Hochschild a inicios de la década de $1980^{41}$. Este emplazamiento a desarrollar lo que en tiempos actuales se ha denominado como empatía apelaba, por lo tanto, a un ejercicio que comprometía (al menos discursivamente) a su protagonista a ir más allá del terreno del mero conocimiento. Así, al hacer referencia a la actitud del orientador para promover actividades como los Consejos de Curso y apoyar la formación de Gobiernos Estudiantiles, se

39 William Reddy, The navigation of feeling. A framework for the history of emotions (Cambridge: Cambridge University Press, 2001), 128.

40 Robert E. Carey, "Métodos de Orientación", en Métodos de Enseñanza en la Educación Secundaria, ed. Harold Spears (Santiago: Programa Interamericano Cooperativo de Educación, 1946), 136. El autor era un importante académico norteamericano que, a la sazón, dirigía el Departamento de Orientación del Servicio de Educación de Nueva York. El subrayado es nuestro.

41 Jan Plamper, The history of emotions, 118-119. 
sostenía que "si todavía, teñimos esos ideales de emoción personal y de auténtica adhesión, los jóvenes captarán nuestras actitudes como estímulos para alcanzar y perfeccionar nuestro sistema democrático"42.

El exigente perfil que se esperaba que cada orientador cumpliera era complementado en una publicación oficial de 1948. Se definía como rasgos constitutivos del cargo que la persona que lo cumpliera tuviera una "personalidad interesante y estimuladora". Se esperaba que su talante estuviera marcado por el "entusiasmo". Debía ser una persona "optimista" y que tuviera "sentido del buen humor". Ahora bien, es importante apreciar que el baremo conductual que se esperaba que cumpliera el orientador se replicaba, con matices, en las exigencias que se le fueron definiendo a los profesores jefes. De tal modo, a ellos se les pedía que desplegaran capacidades afectivas que les permitieran, en sus entrevistas con sus alumnos, "evitar, por todos los medios, que estas conversaciones lleguen a tomar el carácter de una situación fría y estereotipada"43.

En la extensión de las expectativas conductuales y requisitos personales propios de los Consejeros u Orientadores al conjunto de los profesores jefes reside uno de los asuntos operacionales más sensibles en el proceso de instalación de la orientación escolar en el período abordado en estas páginas. Como ya se ha indicado, el contingente de Orientadores era insuficiente en los primeros años. De tal manera, muchos liceos no llegaron a disponer de ellos hasta avanzada la década de 1960. Ello hizo recaer en los profesores jefes parte importante de sus funciones, incluyendo aquellas que suponían cualidades afectivas específicas. Una especialista en el rol de los profesores jefes resumía el desafío de esta manera:

“en países más desarrollados que el nuestro, la administración de los establecimientos educacionales considera en cada profesor los siguientes factores: edad, experiencia, madurez, ideales, iniciativa, originalidad, condiciones de líder, imaginación, paciencia,

42 Jorge Arancibia Jaramillo, "El Gobierno Estudiantil en nuestra Educación Secundaria". Sonap. Revista de la Sociedad Nacional de Profesores. Año I, No2 (1955): 19. El subrayado es nuestro.

43 Dirección General de Educación Secundaria. Departamento Pedagógico. "Circular n²4" 27 de abril de 1954, ALPM, volumen Documentos y Circulares 1953-1954, p.3. 
simpatía, juicio, entusiasmo, tacto y responsabilidad y hace una lista de aquellos que, en atención a estos rasgos, sean aptos para asesores. En nuestro país, dadas las circunstancias en que trabajamos, la gran mayoría de los profesores secundarios debemos aceptar la responsabilidad de ser profesores jefes. La aceptamos, concientes de las limitaciones de nuestra personalidad, pero no podemos olvidar que, dentro de estas limitaciones, debemos estar en continuo afán de superación para no defraudar a nuestros alumnos y no servirles de modelos demasiado imperfectos" ${ }^{\prime 4}$.

En tanto expresión de una regulación institucional, esta enumeración podría entenderse como parte de una naciente emocionología escolar, si se emplea (a guisa de aproximación general) ese concepto fundante de la corriente de la historia de las emociones. Tanto normativas y desiderátums como mirada emocionológica comparten su naturaleza incompleta, en tanto no integran la dimensión de la experiencia, la recepción, adopción, rechazo o resistencia. Esta faceta ignota del proceso es la que hemos reconocido al enfatizar la naturaleza principalmente discursiva del abordaje realizado a la llegada y primeros pasos de la orientación escolar en suelo chileno.

\section{A Modo de Conclusión}

Varios años después de la entrada de los primeros orientadores escolares a los liceos, era posible encontrarse con un colectivo que ya ostentaba un reconocimiento más claro en el sistema educacional chileno, no obstante, que su posición e influencia no lograra prosperar de acuerdo a sus expectativas. Con la fundación en las universidades de la mención de Orientación Escolar en las carreras de Pedagogía, desde fines de la década de 1940 comenzó a surgir una oferta regular de orientadores preparados científicamente para su labor. Siguiendo las dinámicas cambiantes de las décadas siguientes, el discurso sobre la orientación escolar secundaria mantuvo sus desplazamientos oscilantes entre la atención a las demandas de información oportuna para la continuidad laboral o de estudios (siendo esta última cada vez más relevante, conforme el sistema universitario experimentaba una importante expansión) y la atención a las dimensiones conductuales y

44 Radamanta Dintrans de Cáceres, La función del Profesor Jefe. (Santiago: Imprenta Roa, 1956), 14. 
emocionales. En un seminario nacional de orientadores, llevado a cabo en Santiago en 1966 Oscar Fabres, uno de los expositores, advertía a sus colegas que "no debe perderse de vista el principio que la Orientación debe cumplir fundamentalmente una labor profiláctica en el sentido de prevenir los desajustes o problemas de los estudiantes. La Orientación Educacional es fundamentalmente preventiva, lo que no excluye su función terapéutica" ${ }^{45}$. En esta última mención refulgía nuevamente la asociación entre orientación escolar y aspectos psicológicos, la que se veía enfrentada a profundos desafíos en la década de 1960, en la medida que el estallido de la "cuestión juvenil" y las transformaciones culturales causaron una percepción de aceleración del cambio histórico.

Paralelamente a la expansión de la orientación en los liceos chilenos, no exenta de avances y regresiones, la disciplina que le daba su sustento principal (la psicología, especialmente la aplicada a la infancia y juventud) experimentaba dramáticas transformaciones conceptuales que habrían de introducir nuevas tensiones y exigencias a los orientadores. En la medida que deseamos relevar el sentido cada vez más transnacional de los saberes educacionales, es necesario integrar a nuestras reflexiones finales la constatación de que el campo de intervención de la orientación escolar relativo a la comprensión de la naturaleza adolescente debería acometer el uso de nuevos paradigmas. Por ejemplo, la generalización del concepto psicosocial de identidad, a partir de la publicación en 1950 de Childhood and Society, obra de Erik Erikson que reorientó radicalmente los estudios sobre infancia y juventud ${ }^{46}$. La capacidad de integración de este tipo de innovaciones a la formación y práctica de los orientadores escolares es un tema todavía por abordar en la historiografía de la educación chilena. En cualquier caso, entendemos que el análisis de los primeros tiempos de la orientación escolar, de sus episodios expansivos en la década de 1940 o ulteriores transformaciones deben ser sometidas a un doble cedazo: tiempo y naturaleza. Aludiendo a lo primero, es necesario reforzar la idea de que los procesos de reforma educacional se viven en temporalidades no simultáneas ni lineales. El cambio educativo reconoce estratos que poseen

45 Oscar Fabres Villarroel, "La orientación educacional", 2.

46 Nancy. Lesko, Act your age! A cultural construction of adolescence (New York: Routledge, 2012); 121. 
ciertas dosis de autonomía temporal ${ }^{47}$. Y en relación a lo segundo, la índole de disciplinas y conceptos de matriz global o transnacional efectivamente sufre procesos de adaptación y mediación de acuerdo a los contextos loca$\operatorname{les}^{48}$. Ello también deja abierta la interrogante acerca de esas mediaciones realizadas por los orientadores criollos.

Finalmente, cabe preguntarse por la inserción del fenómeno que se ha visitado en estas páginas (la introducción de la orientación escolar en el sistema secundario chileno) dentro de un proceso de más amplio curso. Así, a lo largo del siglo XX ha sucedido una reconfiguración de las relaciones entre los actores de la vida escolar, planteándose que la educación, básicamente en los países de desarrollo avanzado, debe facilitar la autoconstrucción del estudiante como un sujeto expresivo, emprendedor y empoderado, gestor principal de su propia "educación emocional", en una clave de integración funcional al mundo del capitalismo global. Así, en el horizonte de un nuevo siglo emerge, en muy distintas condiciones, la pregunta sobre las posibilidades y pertinencia del rol que ha cumplido durante ya décadas la orientación escolar.

\section{BibLiOgRAFÍA}

\section{a) Fuentes inéditas y archivos}

Archivo del Liceo Enrique Molina Garmendia (ALEMG). Concepción. Archivo del Liceo de Hombres de Puerto Montt (ALPM). Puerto Montt. Archivo del Liceo de Niñas Gabriela Mistral de La Serena (ALNGM). La Serena.

\section{b) Fuentes impresas y bibliografía}

Adam, Thomas. Intercultural transfers and the making of the Modern World. Sources and Contexts.. Londres: Palgrave Macmillan, 2012.

$47 \quad$ Fritz Osterwalder, "Programas educativos, reformas educativas y la longue durée en la historiografía de la educación". en Los sistemas educativos: perspectiva histórica, cultural y sociológica, compiladores Daniel Tröhler y Ragnhild Barbu (Barcelona: Octaedro, 2012), 25.

31248 Una reflexión sobre lo transnacional y procesos de cambio local en Jason Beech, "Continuidades y cambios en el campo educativo global. Influencias externas en la formación docente en Argentina y Brasil", en Internacionalización. Políticas educativas y reflexión pedagógica en un medio global, compiladores Marcelo Caruso y Heinz-Elmar Tenorth (Buenos Aires: Granica, 2011), 183-214. 
Arancibia Jaramillo, Jorge. "El Gobierno Estudiantil en nuestra Educación Secundaria”. Sonap. Revista de la Sociedad Nacional de Profesores. Año I, No2 (1955): 18-23.

Boletín Renovación. Santiago: Ministerio de Educación, 1946.

Carey, Robert E. "Métodos de Orientación”. En Métodos de Enseñanza en la Educación Secundaria, editado por Harold Spears. Santiago: Programa Interamericano Cooperativo de Educación, 1946, 127 138.

Conrad, Sebastian. What is Global History? Princeton: Princeton University Press, 2006.

Dintrans de Cáceres, Radamanta. La función del Profesor Jefe. Santiago: Imprenta Roa, 1956.

Fabres Villarroel, Oscar. "La orientación educacional de la escuela secundaria fiscal". Museo Pedagógico de Chile, Santiago, 3er trimestre (1991): 1-14.

“Ideario del Profesor Orientador". Boletín del Liceo Experimental Manuel de Salas. Año I, no 2 (1944): 55-56.

Iriye, Akira y Pierre-Yves Saunier. The Palgrave Dictionary of Transnational History. Londres: Palgrave Macmillan, 2009.

Iriye, Akira. Global and Transnational History: The Past, Present, and Future. Londres: Palgrave Macmillan, 2013.

Jones, Lawrence K. 'Frank Parsons' contribution to career counseling”. Journal of Career Development, v. 20, n 4, (1994): 287-294.

La renovación del Liceo Chileno. Washington: Publicaciones de la Unión Panamericana, 1945.

Lesko, Nancy. Act your age! A cultural construction of adolescence. New York: Routledge, 2012.

Martin, Jack \& Anne-Marie McLellan. The education of selves: how psychology transformed students. New York: Oxford University Press, 2013.

Mayorga, Rodrigo. Actores transnacionales imperialismo cultural y prácticas discursivas en la irrupción de la sicología educacional en Chile (19301960). Ponencia presentada en ISCHE 2017 (Buenos Aires) Inédito.

Ministerio de Educación. Dirección General de Educación primaria. Instituto de Guía y Orientación Profesional. Exposición Técnica de Orientación Profesional (Guía para el visitante). "Por una Escuela Primaria Mejor". Santiago: sin datos de editorial, 1946. 
Ministerio de Educación Pública. Comisión de Renovación Gradual de la

Educación Secundaria. Disciplina (Adaptada del folleto Disciplina Escolar: de Shriver y Redl, publicado por la Unión Panamericana, Washington, 1944). Santiago: Imprenta y Litografía Stanley, 1947.

Ministerio de Educación Pública. Dirección General de Educación Secundaria. Consejo de Curso y funciones del Profesor Jefe. Santiago: Imprenta Central de Talleres del S.N.S., 1957.

Núñez, Iván. Desarrollo de la Educación Chilena hasta 1973. Santiago: PIIE, 1982.

Núñez Prieto, Iván. "Biología y educación: Los reformadores funcionalistas. Chile, 1931-1948”. Cuadernos Chilenos de Historia de la Educación, nº1 (2013): 65-86.

Osterwalder, Fritz. "Programas educativos, reformas educativas y la longue durée en la historiografía de la educación". En Los sistemas educativos: perspectiva histórica, cultural y sociológica, compiladores Daniel Tröhler y Ragnhild Barbu. Barcelona: Octaedro, 2012,15-27.

Ramírez, Francisco O. y John W Meyer. "Los currículos nacionales: modelos mundiales y legados históricos nacionales". En Internacionalización. Políticas educativas y reflexión pedagógica en un medio global, compiladores Marcelo Caruso y Heinz-Elmar Tenorth. Buenos Aires: Granica, 2011, 107-132.

Reddy, William. The navigation of feeling. A framework for the history of emotions. Cambridge: Cambridge University Press, 2001.

Rocabado Muñoz, Mariano. "El Servicio de Orientación en los liceos chilenos (Comentario sobre las actividades de Orientación Educacional y Vocacional realizadas por los Consejeros durante I947”. En La orientación vocacional en la Enseñanza Secundaria. Santiago: Ministerio de Educación, 1948, 12-24.

Salas, Enrique. "La Orientación Educacional y Vocacional en el Nuevo Liceo". Revista de Educación (1947): 67-71.

Salas, Gonzalo. "Pasado y Presente de la Psicología Científica en Chile: Profesionalización, instituciones y divulgación científica”. En Historias de la Psicología en América del Sur. Diálogos y perspectivas, editado por Gonzalo Salas. La Serena: Nueva Mirada Ediciones, 2014, 100-119.

314 Salas Marchán, Max. “YYo los ayudo!”. Revista de Educación Nacional, Año XX, n³ (1924): 157-162.

Salas Neumann, Emma. Irma Salas. Educación e innovación en Chile. 
Santiago: Corporación Cultural Rector Juvenal Hernández, 2008.

Salas Neumann, Emma. Apuntes sobre educación y algunos retazos. Santiago: sin dato editorial, 2010.

Samper, Armando. Technical cooperation in Latin America. A Case Study

of Cooperation in Secondary Education in Chile. Washington: $\mathrm{Na}$ tional Planning Association, 1957.

Saunier, Pierre-Yves. "Transnational". En The Palgrave Dictionary of Transnational History, editado por Akira Iriye y Pierre-Yves Saunier. Londres: Palgrave Macmillan, 2009, 1047-1054.

Serrano, Sol, Macarena Ponce de León y Francisca Rengifo, (editoras). Historia de la Educación en Chile (1810-2010). Tomo I. Aprender a leer y escribir (1810-1880). Santiago: Taurus, 2012.

Sobe, Noah W. "Researching emotion and affect in the history of education". History of Education: Journal of the History of Education Society, v.41, no5 (2012): 689-695.

Sobe, Noah W. "Entanglement and Transnationalism in the History of American Education". En Rethinking the History of Education. Transnational Perspectives on Its Questions, Methods, and Knowledge, editado por Thomas S. Popkewitz. New York: Palgrave Macmillan, 2013, 93-107.

Stearns Peter y Katherine Stearns. "Emotionology: Clarifying the History of Emotions and Emotional Standards". The American Historical Review, v. 90, no 4 (1985): 813-836.

Toro Blanco, Pablo. "Close to you: building tutorials relationships at the Liceo in Chile in the long 19th century". Jahrbuch für Historische Bildungsforschung 18 (2012): 70-90.

Turbiaux, Marcel. "J.-M. Lahy (1872-1943) et l'orientation professionnelle" Bulletin de psychologie no482/2 (2006): 217-235.

Viñao Frago, Antonio. "La historia de las disciplinas escolares". Historia de la Educación, n²5 (2006): 243-269.

Para citar este artículo: Toro Blanco, Pablo Andrés. "Una nueva oficina en el liceo: la instalación de los orientadores como política educacional en Chile (c.1946-c.1953)", Historia Caribe Vol. XIII No. 33 (Julio-Diciembre 2018): 281-313. DOI: http://dx.doi.org/10.15648/hc.33.2018.11 